\title{
Revista Brasileira de Enfermagem REBEn \\ A enfermagem e a atenção à criança vítima de violência familiar
}

REVISÃo

\author{
The nursing and the attention to the child who is victim of familiar violence
}

La enfermería y la atención al niño victima de violencia familiar

\section{Janice Machado da Cunha}

Enfermeira. Doutoranda em Saúde da Criança no IFF/FIOCRUZ.. Professora Assistente do

Departamento de Enfermagem Materno-Infantil/ FENF/UERJ e pesquisadora do NUSCRIAD (Núcleo de Estudos em Saúde da Criança) jancunha3@yahoo.com.br

Simone Gonçalves de Assis

Médica. Doutora em Ciências e Professora do IFF/FIOCRUZ. Pesquisadora do CLAVES/

FIOCRUZ.

simone@claves.fiocruz.br

Sandra Teixeira de Araújo Pacheco

Enfermeira. Mestre em Enfermagem pela FENF/ UERJ. Professora Assistente do Departamento de Enfermagem Materno Infantil/FENF/UERJ e pesquisadora do NUSCRIAD - E-Mail: stapacheco@aol.com

\section{RESUMO}

Estudo bibliográfico com o objetivo de refletir sobre a atenção de enfermagem à criança vítima de violência familiar a partir da análise da produção científica acerca desta temática. Foram analisadas 37 publicações (08 nacionais e 29 internacionais) indexadas (BDENF; LILACS e MEDLINE), localizadas no período de 1993-2003. A utilização da técnica de análise de conteúdo possibilitou a categorização do conhecimento em três núcleos temáticos: diagnóstico, capacitação do enfermeiro e atenção de enfermagem à criança vítima de violência familiar. Não se visualizou um crescimento significativo da produção científica da Enfermagem enfocando este tema nos últimos anos.

Descritores: Violência doméstica; Criança; Enfermagem pediátrica.

\section{ABSTRACT}

Bibliographic study that aimed to reflect about the nursing care to the child who is victim of familiar violence from the analysis of scientific production concerning this subject. Thirty-seven indexed publications were analyzed (08 national and 29 international) using the following bibliographic databases: BDENF; LILACS and MEDLINE. Estalishing the period of 1993-2003 as limit time. The results were analyzed applying the technique of content analysis becoming possible the distinction of knowledge in three topical cores: diagnostic, qualification of the nurse, and nursing care to the child victim of familiar violence. An increase of significant nursing scientific production focalizing this subject was not visualized in the last years.

Descriptors: Domestic violence; Child; Pediatric nursing.

\section{RESUMEN}

Estúdio bibliográfico con el objectivo de reflexionar sobre la atención de enfermería al nino víctima de violência familiar a partir del análisis de la produccion científica referente a esa temática. Fueron analisadas 37 publicaciones (08 nacionales y 29 internacionales) indexadas (BDENF, LILACS Y MEDLINE), sitas em nel período de 1993-2003. Los datos fueron analisados por médio de la técnica de análises de contenido, lo que propició la categorización del conocimiento in três núcleos temáticos: diagnóstico, capacitación del enfermero y atención de enfermería al nino víctima de violência familiar. Non se noto um crecimiento significativo de la producción científica de la enfermeria tratando de ese tema em los últimos años.

Descriptores: Violência familiar; Niño; Enfermería pediátrica.

Cunha JM, Assis SG, Pacheco STA. A enfermagem e a atenção à criança vítima de violência familiar. Rev Bras Enferm 2005 jul-ago; 58(4):462-5.

\section{INTRODUÇÃO}

O enfrentamento da violência e suas conseqüências têm sido um desafio para os profissionais de saúde, embora não seja um problema específico desta área. As crianças estão incluídas entre os grupos humanos mais vulneráveis aos eventos violentos e muitas vezes estas situações ocorrem no contexto familiar, caracterizando-se como um problema de grande relevância social e científica.

Algumas pesquisas nacionais têm possibilitado antever a elevada prevalência de violência familiar na infância. Um estudo que permite evidenciar esta realidade foi realizado em $1990^{(1)} \mathrm{com} 1328$ adolescentes matriculados em escolas num Município do Estado do Rio de Janeiro. Este trabalho constatou que $52,8 \%$ dos adolescentes sofriam violência física de um ou de ambos os pais. Entre as práticas violentas citadas destacaram-se: tapas, bofetões, tentar bater ou bater com objetos, ameaçar ou ferir com armas. Outro estudo (2) mais recente, investigou 1685 adolescentes estudantes de escolas públicas e particulares de São Gonçalo/RJ, constatando que 14,6\% dos entrevistados sofrem violência física severa de pai ou mãe (atos como chutar, morder ou dar murros, espancar, ameaçar ou efetivamente usar arma de fogo ou arma branca); $11,8 \%$ testemunharam ou vivenciaram 
violência sexual na família; 48\% relataram sofrer violência psicológica de pessoas significativas.

Tem crescido no Brasil, especialmente na área da saúde, a necessidade de uma reflexão mais profunda sobre este problema em decorrência dos novos paradigmas colocados pelo Estatuto da Criança e do Adolescente (ECA), em 1990(3). Esta Lei, entre outras determinações, estabelece a obrigatoriedade de notificação dos casos suspeitos ou confirmados de maus-tratos contra a criança e institui uma penalidade para os profissionais de saúde ou educação que não atenderem a esta determinação.

Embora se reconheça a importância da multidisciplinaridade na atenção à criança vítima de violência familiar, considera-se que a Enfermagem, e mais especificamente o Enfermeiro Pediatra têm um papel importante neste processo. Este pressuposto baseia-se na constatação de que o Enfermeiro tem como foco principal a assistência direta e integral ao cliente, além de ser um dos profissionais que permanece por maior período convivendo com a criança e sua família seja no contexto hospitalar, em unidade básica de saúde ou em ambiente familiar/comunitário.

Nesta perspectiva, este artigo tem como objetivo refletir sobre a atenção de enfermagem à criança vítima de violência familiar, a partir da análise da produção científica da enfermagem nacional e internacional acerca desta temática.

Não obstante a multiplicidade de definições e conceitos, neste estudo entende-se por violência familiar contra a criança ações e/ou omissões perpetradas por parentes ou responsáveis pela criança, podendo causar danos físicos, sexuais e/ou psicológicos às pequenas vítimas.

\section{CONSIDERAÇÕES METODOLÓGICAS}

Trata-se de um estudo bibliográfico, tendo sido adotado os seguintes procedimentos para levantamento e análise da documentação bibliográfica: busca, seleção, impressão/solicitação e análise dos textos. Através do site da Biblioteca Regional de Medicina (BIREME), realizouse um levantamento da literatura científica indexada nas bases de dados: MEDlars Online Literatura Internacional (Medline), Literatura LatinoAmericana e do Caribe (LILACS) e Banco de Dados da Enfermagem (BDENF). Foram utilizados os seguintes descritores em português: "violência doméstica", "violência familiar", "maus tratos", "enfermagem" e "criança". Em inglês: "child abuse", "neglect", "nursing", "family" e "violence". O universo das definições de violência familiar foi muito diversificado, constituindo-se num dos maiores desafios deste estudo. Optou-se por considerar como sinônimos os descritores relativos à violência. Inicialmente foram encontradas as seguintes freqüências de produções científicas: 92 (Medline), 15 (LILACS) e 12 (BDENF).

Os critérios adotados para exclusão no estudo foram: produção científica de Enfermeiro ou de outros profissionais que não incluíssem a atenção de enfermagem à criança vítima de violência familiar, estudos sobre violência contra outros grupos humanos sem enfoque na infância e referências bibliográficas incompletas ou repetidas.

Seguindo os critérios de inclusão adotados neste estudo foram selecionadas 37 produções científicas que serviram de base para a análise, no entanto estarão sendo referenciadas ao longo deste texto, aquelas consideradas de maior relevância para o presente artigo.

Tem-se a clareza de que esta pesquisa não contempla todas as publicações brasileiras e internacionais sobre a temática. Contudo, a análise de dos textos nacionais e internacionais possibilitou traçar um panorama aprofundado e extensivo da temática e mapear o estado da arte da produção científica da Enfermagem acerca da atenção à criança vítima de violência familiar.

A análise dos dados pautou-se na associação das abordagens quantitativa e qualitativa, utilizando-se a técnica de análise de conteúdo, na modalidade temática. Após seleção dos textos, procedeu-se a leitura flutuante e organizou-se o corpus de análise de acordo com os objetivos da pesquisa. Após leituras exaustivas foram identificadas as unidades de registro que a seguir foram agrupadas em núcleos temáticos ${ }^{(4)}$ que emergiram de forma de isolada ou associada nas publicações.

\section{RESULTADOS E DISCUSSÃO}

3.1 Panorama da produção de conhecimento acerca da atenção de Enfermagem à criança maltratada por seus familiares

Num total de 37 publicações, sendo 08 (21,6\%) nacionais e 29 $(78,4 \%)$ internacionais. Constatou-se a predominância das produções internacionais, majoritariamente procedentes dos Estados Unidos (24), seguidos da Inglaterra (04) e Escócia (01). As publicações brasileiras tiveram como origem os seguintes estados: São Paulo(03), do Rio Grande do Sul (02), Bahia(02) e Santa Catarina(01).

Quanto à metodologia adotada, na produção internacional houve maior freqüência dos artigos de reflexão teórica (13), sendo as demais publicações categorizadas como: estudos quantitativos (8), revisões bibliográficas (06), relato de experiência (01) e qualitativo (01). A distribuição dos textos nacionais segundo o enfoque metodológico foi: estudos quantitativos (03), artigos de reflexão teórica (02), revisão bibliográfica (01), abordagem qualitativa (01) e quanti-qualitativo (01).

No que se refere à distribuição da freqüência das produções científicas nos últimos 10 anos, não se visualizou um aumento significativo do número de publicações: O período 1996-2000 concentra a maior parte das publicações, sendo 15 internacionais e 04 nacionais; no período 1993-1995 foram encontrados 5 artigos internacionais e 03 nacionais e no período 2001-2003 houveram 9 publicações internacionais e apenas 1 artigo em revista nacional.

Os resultados deste estudo no âmbito nacional, foram semelhantes aos dados obtidos em estudos bibliográficos anteriores ${ }^{(5-6)}$ que localizaram um número pequeno de produções científicas brasileiras com enfoque na Enfermagem e a atenção à violência familiar na infância. Uma pesquisa bibliográfica ${ }^{(5)}$ acerca das propostas de prevenção à violência, constatou que num universo de 48 produções científicas, apenas 2,1\% eram da Enfermagem. Outro estudo ${ }^{(6)}$, num total de 105 trabalhos encontrou apenas sete produções de Enfermeiros. Isto é preocupante, pois considerando a exposição da temática na mídia, os dados relativos à epidemiologia da violência e os aspectos jurídicos que comprometem os profissionais de saúde com este problema de saúde pública, uma repercussão maior no meio acadêmico seria esperada.

Os temas enfocados no corpus de análise deste estudo apresentaram uma grande heterogeneidade. A bibliografia internacional no que se refere às vítimas da violência destacou de forma associada os idosos, as mulheres e as crianças como os mais freqüentemente vitimizados. Os textos nacionais enfatizaram a criança como vítima e a perspectiva da atenção extensiva a toda a família.

A análise da produção científica possibilitou a constituição de três núcleos temáticos: a) atuação do enfermeiro frente aos casos de crianças vítimas de violência familiar, enfatizada em 23 (79,3\%) dos trabalhos internacionais e $4(50 \%)$ das publicações em âmbito nacional ; b) 0 diagnóstico da violência familiar contra a criança foi enfocado em 21 $(72,4 \%)$ textos internacionais e $6(75 \%)$ nacionais e c) a capacitação do enfermeiro para atender à crianças vítimas de maus tratos familiares foi abordada em $11(37,9 \%)$ estudos internacionais e $3(37,5 \%)$ nacionais. Como alguns trabalhos abordaram vários temas, o total excede o número de trabalhos avaliados.

3.20 diagnóstico: primeiro passo na atenção à crianças vítimas de violência familiar

Constatou-se que as produções nacionais e internacionais enfatizaram a importância do diagnóstico como um primeiro passo na atenção de enfermagem à criança vítima de violência familiar. Para 0 
diagnóstico do problema considera-se fundamental o reconhecimento e a definição do que se entende por violência. Os textos internacionais e nacionais de certa forma foram convergentes ao caracterizar a violência como um problema complexo, multi-fatorial e que afeta a saúde individual e coletiva ${ }^{(7-13)}$. Devido ao caráter multidimensional, há o reconhecimento que $a$ atenção às criança vítima de violência envolve diferentes setores da sociedade. Contudo, não se visualizou um consenso no que se refere à definição da temática, predominando uma certa indefinição e multiplicidade do que se considera violência familiar contra a criança.

Outro aspecto importante no reconhecimento da vitimização familiar contra a criança é a demonstração da magnitude deste problema. Embora, a maioria das publicações ressalte a relevância da temática constatou-se que os textos norte-americanos apresentaram estatísticas oficiais relativas à prevalência de violência familiar(12,14-17). Nos textos nacionais os dados estatísticos, quando apresentados se referiram a estudos de prevalência restritos a determinada localidade ou região( ${ }^{(8,18)}$

Ao demonstrar a relevância da atenção de enfermagem à vitimização infantil no contexto familiar, muitas publicações alertaram para as conseqüências deste problema no desenvolvimento bio-psico-social das crianças. O impacto psicológico foi ressaltado como um dos mais preocupantes uma vez que as crianças, por estarem ainda em processo de desenvolvimento, são mais vulneráveis à influência dos adultos ${ }^{(7,11,19}$

${ }^{21)}$. Entre as respostas psicológicas, foram descritas: estresse, ansiedade e preocupação. Sendo analisado que o processo de vitimização pode levar a criança a não interagir socialmente, o que muitas vezes pode ser um fator que dificulta o diagnóstico e em outras vezes pode ser caracterizado como um indicador de violência, facilitando assim a identificação de situações de sofrimento infantil, por parte do profissional. Este processo está estritamente relacionado à capacitação do profissional no reconhecimento dos indicadores de cada tipo de abuso.

As publicações abordaram de forma associada ou isolada, diferentes modalidades de violência familiar contra a criança, predominando os textos sobre abuso físico e sexual. A violência psicológica também foi muito destacada, sendo mais freqüentemente descrita como associada a outras modalidades de violência. A negligência foi o tipo de violência menos enfocado na bibliografia internacional, todavia alguns autores brasileiros ${ }^{(7)}$ estudaram de forma bastante aprofundada esta modalidade de violência.

No que se refere ao diagnóstico dos casos de crianças vitimizadas, nas publicações internacionais, constatou-se um esforço na utilização de indicadores, roteiros e escalas para reconhecer e classificar os sinais de violência ${ }^{(10,14)}$. Alguns estudos tiveram como objetivo a avaliação da capacidade dos profissionais na identificação dos casos de violência, geralmente utilizando instrumentos de avaliação que mensuravam os conhecimentos pré e pós treinamentos realizados ${ }^{(14,22)}$. Nos textos nacionais, a ênfase maior foi na sensibilização dos profissionais para a problemática: um estudo apresenta roteiro de identificação de $\operatorname{casos}^{(20)}$, 5 trabalhos pontuam o papel do enfermeiro ${ }^{(7,9,19,20,23)}$ e 2 textos buscaram caracterizar o problema através de estudos de prevalência de forma a subsidiar a ação da enfermagem ${ }^{(8,18)}$. Apontam, portanto para a necessidade de estudos nacionais que possam fundamentar e preparar os enfermeiros para identificar e atender a criança vítima de violência familiar contra a criança.

\subsection{O Enfermeiro está capacitado para cuidar de crianças} vítimas de violência familiar?

Embora este tema tenha sido o menos abordado nos textos analisados, sua relevância mostra-se fundamental para o enfrentamento dos maus-tratos na infância pelo setor de saúde.

A importância da capacitação do Enfermeiro foi apontada por alguns autores, assinalando a relevância da inclusão da temática no currículo. Na produção científica internacional, este tema foi abordado em alguns estudos de forma sistematizada, relatando experiências de currículo de enfermagem que contemplaram a temática ${ }^{(16,24,25)}$. Foram publicados também relatos de experiências de capacitação de profissionais já formados sendo destacados aspectos como: o conhecimento dos fatores associados aos maus-tratos, as conseqüências da violência, o sistema de proteção legal e os recursos da comunidade que podem contribuir para a prática de enfermagem ${ }^{(26)}$.

No âmbito nacional, alguns textos enfocaram a importância da inserção da temática nos currículos ${ }^{(7,9,20,23)}$, isto sinaliza a necessidade do aprofundamento da discussão desta questão nas associações de classe e instituições responsáveis pela formação dos enfermeiros.

\subsection{A atenção à criança vítima de violência familiar: um desafio para a Enfermagem}

O tema mais destacado nos textos foi a atenção às vítimas, tendo a perspectiva da atuação multidisciplinar norteado vários estudos. Contudo, a Enfermagem foi apontada nas produções nacionais ${ }^{(7,9,19,20,23)}$ e internacionais ${ }^{(11,12,17,27)}$, como uma categoria profissional com um papel fundamental na identificação dos casos, no tratamento e proteção das vítimas.

Não obstante, a prioridade dos autores deste artigo tenha sido a atuação dos enfermeiros da área pediátrica, a análise das publicações nacionais e internacionais possibilitou a identificação de outras especialidades da Enfermagem com ação direta no atendimento às vítimas de violência. Além da pediatria, foram relacionadas áreas de saúde pública, saúde mental e emergência. Na bibliografia internacional destaca-se ainda a área do atendimento domiciliar, considerada prioritária tanto na prevenção da violência, quanto no acompanhamento e tratamento dos casos após a identificação. Outro aspecto ressaltado na produção internacional e ausente na bibliografia nacional foi, a importância de profissionais de enfermagem sem formação superior atuando frente aos maus-tratos. Um estudo ${ }^{(12)}$ aponta que profissionais de enfermagem com vários níveis de educação e escolaridade podem realizar ações como: identificação de casos, intervenções em crises e terapia físicas.

A atenção nos diferentes níveis de complexidade foi amplamente abordada, mas muitos autores priorizaram as ações de prevenção analisando a importância de atividades como: orientação aos pais quanto aos cuidados com as crianças pequenas, programas de educação preventiva nas escolas e diagnóstico precoce através de visitas domiciliares ${ }^{(17,27,28,29)}$.

A história de vida, a postura profissional e as atitudes pessoais dos profissionais de enfermagem foram apontadas como aspectos que interferem na atuação frente aos casos de crianças vitimizadas por suas famílias $^{(19,20,23)}$. Enfatizou-se a necessidade dos Enfermeiros terem uma atitude baseada na sensibilidade, capacidade de escuta e compreensão. Nesta perspectiva ressaltam-se alguns textos brasileiros que abordam com muita propriedade o impacto da violência na vida de enfermeiros que cuidam de crianças vitimizadas ${ }^{(7,9,19,23)}$, demonstrando o quanto são conflitantes as atitudes idealizadas e as concretizadas.

Neste sentido, considera-se fundamental a discussão acerca dos dilemas ético-legais na atenção às crianças vitimizadas no contexto familiar, destacando-se os decorrentes da obrigatoriedade de notificação dos casos às autoridades judiciais e os inerentes à assistência de enfermagem orientada pelos princípios éticos da beneficiência, autonomia e justiça ${ }^{(7,9,11,13,20)}$.

\section{CONSIDERAÇÕES FINAIS}

Neste estudo constatou-se que a produção científica da Enfermagem assinala a relevância da atuação dos Enfermeiros nos diferentes níveis da atenção à criança vítima de violência familiar, com especial destaque para as ações preventivas. Esta atuação prioriza o cuidado à criança $e$ sua família, considerando-se a violência como um problema multidi- 
mensional e que para enfrentá-lo é necessário capacitação e trabalho em equipe.

O universo das abordagens sobre violência foi muito heterogêneo, sendo difícil apontar uma única tendência na produção científica acerca da temática. Entre os estudos nacionais predominaram as pesquisas relacionadas a sensibilização da enfermagem para a problemática e no âmbito internacional um dos temas mais focalizados foi a importância da enfermagem no diagnóstico precoce dos casos de violência familiar contra criança.

\section{REFERÊNCIAS}

1. Assis SG. Violência doméstica. Estudo epidemiológico sobre a vitimização física dos adolescentes de Duque de Caxias. Med Ribeirão Preto 1995; 28(1):51-60.

2. Assis SG, Avanci JQ. Labirinto de espelhos. A formação da autoestima na infância e adolescência. Rio de Janeiro (RJ): Editora Fiocruz; 2004.

3. Brasil. Ministério da Justiça. Estatuto da Criança e do Adolescente. Lei no. 8069 de 13 de julho de 1990 . Brasília (DF). 1990.

4. Minayo MCS. O desafio do conhecimento: pesquisa qualitativa em saúde. São Paulo (SP): HUCITEC / Rio de Janeiro (RJ): ABRASCO 1996.

5. Gomes R, Silva CMFP, Njaine K. Prevenção à violência contra a criança e o adolescente sob a ótica da saúde: um estudo bibliográfico. Ciência e Saúde Coletiva 1999; 4(1): 171-81.

6. Assis SG, Constantino P. Violência contra crianças e adolescentes: o grande investimento da comunidade acadêmica na década de 90 In: Minayo MCS, Souza ER, organizadores. Violência sob o olhar da Saúde: a infrapolítica da contemporaneidade brasileira. Rio de Janeiro (RJ): Editora FIOCRUZ; 2003. p.163-98.

7. Morais EP, Eidit OR. Conhecendo para evitar: a negligência nos cuidados de saúde com crianças e adolescentes. Rev Gaúcha Enferm 1999; 20 (esp): 6-21.

8. Santos NOD. Abuso sexual: vítimas das relações familiares. Rev Baiana Enferm 1995; 8(1/2):21-36

9. Ferriani MC, Ceribelli C, Neves FRAL, Cano MAT, Ubeda EML. Crianças e adolescentes vítimas de violência doméstica: a enfermagem neste cenário. Acta Paul Enferm 2001; 14(2):46-54

10. Little L, Kantor GK. Using ecological theory to understand intimate partner violence and child maltreatment. J Community Health Nurs 2002; 19(3):133-45.

11. Draucker CB. Domestic violence: the challenge for nursing. Online J Issues Nurs 2002; 7(2):13.

12. Merrell J. Social suport for victims of domestic violence. J Psychosoc Nurs Ment Health Serv 2001; 39(11): 30-5.

13. Freed PE ; Drake VK Mandatory reporting of abuse: pratical, moral and legal for psichiatric home healthcare nurses. J Psychosoc Nurs Ment Health Serv 1999; 37:423-436.

14. Spencer D. Paediatric trauma: when it is not an accident. Accid Emerg Nurs 2002; 10(3):143-8.

15. Thobaben M. Caring for interpersonal violence survivors. Home Care
Mesmo reconhecendo que este estudo não abarcou a totalidade da produção científica sobre a temática, considera-se a produção científica nacional ainda incipiente, sendo fundamental aprofundar a reflexão visando a produção de mais estudos da Enfermagem sobre a sua atuação na atenção à criança vítima de violência familiar. Chama atenção o pequeno crescimento da produção na área de enfermagem, face ao grande investimento que está havendo na área da atenção às crianças vítimas de violência no Brasil.

16. Woodtli MA, Breslin E. Violence-related content in the nursing curiculum: a national study. J Nurs Educ 1996; 35(8):367-74.

17. Campbell JC, Harris MJ, Lee RK. Violence resarch: an overview. Sch Inq Nurs Pract1995; 9(2):105-26.

18. Camargo $\mathrm{CL}$. Violência física familiar contra criança e adolescentes: um recorte localizado [tese]. São Paulo (SP): Faculdade de Saúde Pública,Universidade de São Paulo; 1996.

19. Eidt OR, Biehl J, Algeri S. Atelier de vivências: um ambiente propício à construção do cuidado à criança hospitalizada por maus- tratos. Rev Gaúcha Enferm 1998; 19(1):47-55.

20. Brêtas JRS, Silva CV, Quirino MD, Ribeiro CA, Kurasima AY, Meira AOS. $O$ enfermeiro frente à criança vitimizada. Acta Paul Enferm 1994; 7(1):3-10.

21. Attala J, McSweeney M. Preschool children of battered women identified in a community setting Issues. Compr Pediatr Nurs 1997; 20(4):217-25.

22. Berger RP, Bogen D, Dulani T, Broussard E. Implementation of a program to teach pediatric residents and faculty about violence. Arch Pediatr Adolesc Med 2002; 156(8):804-10.

23. Biehl Jl. Criança hospitalizada por maus-tratos: 0 cuidado e 0 significado das vivências de cuidadores de enfermagem [dissertação]. Florianópolis (SC): Departamento de Enfermagem, Universidade Federal de Santa Catarina; 1997.

24. Ross MM, Hoff LA. Coutu-Wakulczyk G. Nursing curricula and violence issues. J Nus Educ 1998; 37(2):5-60.

25. Mandt AK. The curriculum revolution in action: nursing and crisis intervention for victms of family violence. J Nurs Educ 1993; 32(1):44-6.

26. Parsons $\mathrm{LH}$, Moore ML Family violence issues in obstetrics and ginecology, primary care, and nursing texts. Obstet Gynecology 1998; 90(4 Pt 1):158-9.

27. Peckover S. Focusing upon children and men in situations of domestic violence: an anlysis of the gendered nature of British health visiting. Health Soc Care Community 2002; 10(4):254-61.

28. Häggman-Laitila A. Early support needs of Finnish with small children. J Adv Nurs 2003; 41(6):595-606.

29. Eckenrode J, Ganzel B, Henderson CR, Smith E, Olds DL, Powers $\mathrm{J}$, et al. Preventing child abuse and neglet with a program of nurse home visitation: the limiting effects of domestic violence. JAMA 2000; 284(11):1385-91. 